\title{
Direct identification of base-paired RNA nucleotides by correlated chemical probing
}

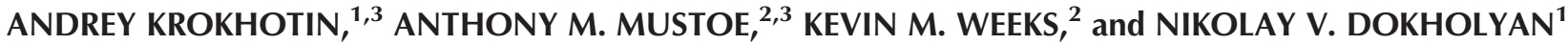 \\ ${ }^{1}$ Department of Biochemistry and Biophysics, ${ }^{2}$ Department of Chemistry, University of North Carolina at Chapel Hill, Chapel Hill, North Carolina \\ 27599, USA
}

\begin{abstract}
Many RNA molecules fold into complex secondary and tertiary structures that play critical roles in biological function. Among the best-established methods for examining RNA structure are chemical probing experiments, which can report on local nucleotide structure in a concise and extensible manner. While probing data are highly useful for inferring overall RNA secondary structure, these data do not directly measure through-space base-pairing interactions. We recently introduced an approach for singlemolecule correlated chemical probing with dimethyl sulfate (DMS) that measures RNA interaction groups by mutational profiling (RING-MaP). RING-MaP experiments reveal diverse through-space interactions corresponding to both secondary and tertiary structure. Here we develop a framework for using RING-MaP data to directly and robustly identify canonical base pairs in RNA. When applied to three representative RNAs, this framework identified $20 \%-50 \%$ of accepted base pairs with a $<\mathbf{1 0} \%$ false discovery rate, allowing detection of $\mathbf{8 8} \%$ of duplexes containing four or more base pairs, including pseudoknotted pairs. We further show that base pairs determined from RING-MaP analysis significantly improve secondary structure modeling. RING-MaP-based correlated chemical probing represents a direct, experimentally concise, and accurate approach for detection of individual base pairs and helices and should greatly facilitate structure modeling for complex RNAs.
\end{abstract}

Keywords: RNA secondary structure; chemical probing; pseudoknots; structure prediction; DMS reactivity

\section{INTRODUCTION}

RNA molecules play diverse and central roles in coordinating gene expression, ranging from regulating transcription, splicing, and translation, to directing cellular localization and RNA turnover. These regulatory functions are critically dependent on the ability of RNAs to fold back on themselves to form complex secondary structures (Cech and Steitz 2014; Mortimer et al. 2014). Accurately determining the set of Watson-Crick base pairs that define RNA secondary structure is therefore fundamental to understanding RNA biological function and is also an essential first step in defining global tertiary structure (Hajdin et al. 2010; Bailor et al. 2011; Magnus et al. 2014; Krokhotin et al. 2015).

In general, correctly defining RNA secondary structure requires experimental information. Sequence covariation analysis remains the gold standard for secondary structure modeling but can be applied to only a very limited set of RNAs (Seetin and Mathews 2012). Computational approaches based on optimization of empirical free energy functions (Zuker 2003; Reuter and Mathews 2010; Lorenz et al. 2011)

${ }^{3}$ These authors contributed equally to this work.

Corresponding authors: weeks@unc.edu, dokh@unc.edu

Article is online at http://www.rnajournal.org/cgi/doi/10.1261/rna.058586. 116 work well for RNAs shorter than roughly $50 \mathrm{nt}$, but have limited accuracy for longer and complexly folded RNAs. In addition, experiment-free approaches are insensitive to functionally important changes in structure induced by varied solution conditions or binding by small molecule or macromolecular ligands.

Chemical probing experiments are among the most powerful and widely applicable approaches for directly interrogating the structure of an RNA molecule. The most useful reagents react specifically with single-stranded or conformationally flexible nucleotides. Nucleotide-resolution reactivity information can then be used to guide computational structure modeling toward a single secondary structure or collection of structures most compatible with the experimental data (Deigan et al. 2009; Weeks 2010). Methods based on these one-dimensional probing data are quite powerful and have been used to derive the structures of a variety of RNAs with good-to-excellent accuracy (Hajdin et al. 2013; Leonard et al. 2013; Siegfried et al. 2014). Data from multiple

\footnotetext{
(C) 2016 Krokhotin et al. This article is distributed exclusively by the RNA Society for the first 12 months after the full-issue publication date (see http://rnajournal.cshlp.org/site/misc/terms.xhtml). After 12 months, it is available under a Creative Commons License (Attribution-NonCommercial 4.0 International), as described at http://creativecommons.org/licenses/by$\mathrm{nc} / 4.0 \%$.
} 
reagents with complementary structural specificities can also be combined to achieve increased modeling accuracy (Rice et al. 2014; Sloma and Mathews 2015).

A key limitation, however, is that secondary structures derived from one-dimensional probing data are not visualized directly, but rather are inferred based on compatibility with the observed reactivity profile. Sometimes, multiple basepairing patterns are nearly equally compatible with structure-probing data. Individual helices and base pairs can be confirmed by repeating probing experiments on RNAs containing point mutations (Duncan and Weeks 2008; Kladwang and Das 2010), but these mutate-and-probe approaches are laborious and not widely pursued. Diverse innovative approaches that seek to directly identify RNA duplexes using massively parallel sequencing have been recently proposed (Weidmann et al. 2016). Validation of these approaches is ongoing, but it is likely that biases from experimental and sequencing-library preparation steps will limit these methods to qualitative structure mapping (Weeks 2015). Thus, there currently exists a tension between experimental tractability versus accuracy and confidence in the derived structure models.

Recently, we developed a new chemical probing strategy termed mutational profiling $(\mathrm{MaP})$ that represents an important experimental and conceptual advance in nucleic acid structure probing. In MaP, chemically modified nucleotides are detected when a reverse transcriptase reads through the modification site while simultaneously incorporating a noncomplementary nucleotide or causing a deletion in the synthesized cDNA (Siegfried et al. 2014). Modification sites are thus recorded as internal sequence changes that can be efficiently read out by massively parallel sequencing. Importantly, this strategy allows for the detection of multiple chemical modifications in a single RNA molecule, and therefore detection of correlated modification events that report on RNA interaction groups, or RINGs (Homan et al. 2014). In initial proof-of-principle studies using the reagent dimethyl sulfate (DMS), RING-MaP experiments were shown to detect diverse through-space secondary and tertiary structure interactions in RNA (Homan et al. 2014).

Here we adapt the RING-MaP strategy to enable comprehensive, direct identification of Watson-Crick base pairs. In our original RING-MaP study, analysis was restricted to adenosine (A) and cytidine (C) nucleotides, which were previously thought to be the only nucleotides with readily detectable DMS modification. In addition, RING-MaP experiments yield very dense networks of interconnected RINGs that are challenging to interpret, precluding straightforward assignment of individual RINGs to secondary versus tertiary structure interactions. The density of interactions concealed the potential utility of RING-MaP experiments for de novo secondary-structure determination. We show for the first time that DMS treatment consistently yields detectable modifications at guanosine $(\mathrm{G})$ and uridine $(\mathrm{U})$ nucleotides. Through correlation analysis and development of a novel filtering algorithm, we then demonstrate that RING-MaP data can be used to directly and specifically detect Watson-Crick base-pairing interactions, facilitating de novo RNA structure modeling.

\section{RESULTS}

\section{DMS reacts with all four RNA nucleotides}

DMS is a classic and widely used RNA structure-probing reagent that is conventionally used to monitor the pairing status of A and C nucleotides (Peattie and Gilbert 1980). In particular, DMS reacts to form methyl adducts at solventaccessible $\mathrm{N} 1$ and $\mathrm{N} 3$ positions on the Watson-Crick faces of $\mathrm{A}$ and $\mathrm{C}$ nucleotides, respectively. These adducts can be readily detected by reverse-transcription analysis, as the modification at the Watson-Crick face induces premature cDNA termination or, in the MaP strategy, incorporation of a noncomplementary nucleotide. While generally ignored, DMS also is known to methylate both $G$ and $U$ residues (Lawley and Shah 1972; Kuśmierek and Singer 1976; Chang and Lee 1981; Ashworth et al. 1985). For example, DMS readily reacts with the N7 of the imidazole ring of G; however, N7 adducts do not efficiently interfere with conventional reverse transcription and are thus not typically detected in probing experiments (Ehresmann et al. 1987). G nucleotides can also be methylated at the $\mathrm{N} 1$ and $\mathrm{N} 3$ positions, and $\mathrm{U}$ nucleotides can be methylated at the N3 position, likely through mechanisms that involve transient base deprotonation or tautomerization (Lawley and Shah 1972; Kuśmierek and Singer 1976; Chang and Lee 1981; Ashworth et al. 1985). These modifications are at the Watson-Crick face and thus should be detectable by reverse-transcription analysis. Indeed, DMS-reactive $U$ and G positions are occasionally observed (Konforti et al. 1998; Bayfield et al. 2001). However, for most $\mathrm{G}$ and $\mathrm{U}$ nucleotides, the modification rate of $\mathrm{N} 1$ and N3 positions is below the detection sensitivity of traditional termination-based reverse-transcription analysis.

The MaP strategy offers significantly greater detection sensitivity compared to traditional termination-based approaches, allowing accurate measurement of nucleotide modifications that occur only a fractional percentage of the time. In reexamining our original RING-MaP data collected on the Escherichia coli thiamine pyrophosphate (TPP) riboswitch, the P546 domain of the T. thermophila group I intron, and the catalytic domain of B. stearothermophilus RNase P (Homan et al. 2014), we observed DMS-dependent mutation signals at $G$ and $U$. The rates of these mutations were considerably lower than those of A and C but were consistent with the signal expected from DMS modification. The mutations observed at $\mathrm{U}$ nucleotides likely reflect modification at $\mathrm{N} 3$, whereas mutations at $\mathrm{G}$ nucleotides likely reflect a combination of N1 and N3 methylations and partial detection of N7 methylation. 

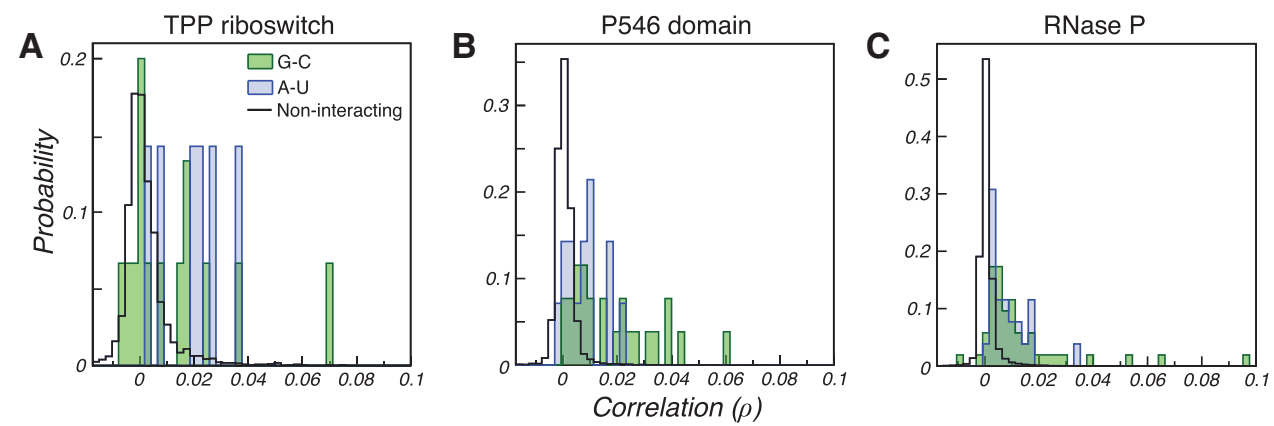

FIGURE 1. Distribution of correlation coefficients for the (A) TPP riboswitch, $(B)$ P546 domain, and $(C)$ RNase P catalytic domain RNAs. Correlations between true $\mathrm{G}-\mathrm{C}$ and $\mathrm{A}-\mathrm{U}$ base pairs versus random combinations of noninteracting $\mathrm{A}-\mathrm{U}$ and $\mathrm{G}-\mathrm{C}$ nucleotides are shown.

\section{Watson-Crick base pairs exhibit strong RINGs}

Given the success of the RING-MaP approach at detecting direct through-space interactions in RNA (Homan et al. 2014), we examined whether RING-MaP data could be used to comprehensively identify Watson-Crick base pairs. While Watson-Crick-paired nucleotides are protected from DMS modification at equilibrium, transient base-pair melting should expose paired nucleotides to DMS modification in a concerted manner, giving rise to correlated modifications measurable via RING-MaP. DMS adducts also destabilize base pairs, and thus chance modification of one nucleotide in a base pair will directly increase the probability that the partner nucleotide is subsequently modified. Lowly populated alternative conformations and unfolded states may also expose paired nucleotides to modification in a concerted manner.

Supporting the hypothesis that base-paired nucleotides undergo correlated DMS modification, we observe distinctly stronger correlated mutations between Watson-Crick-paired nucleotides compared to random (noninteracting) $\mathrm{A}-\mathrm{U}$ and $\mathrm{C}-\mathrm{G}$ nucleotides (Fig. 1). G-C base-paired nucleotides exhibit moderately stronger correlations, on average, than A-U base-paired nucleotides, likely reflecting the lower modification rate of $U$ nucleotides compared to $G$ nucleotides. Together, this analysis indicates that Watson-Crick pairs give rise to detectable correlated mutations (RINGs), suggesting that RING-MaP data could facilitate direct visualization of RNA secondary structure.

\section{Base-pair detection using filtered correlation analysis}

In order to be useful, de novo structure determination approaches must satisfy two criteria: The method (i) must possess high sensitivity and thereby identify a usefully large fraction of true base pairs, and (ii) must minimize the number of potentially misleading false-positive predictions. It is clear that strong RING correlations are indicative of true base pairs (Fig. 1). Thus, a straightforward strategy for specifically identifying base pairs would be to search for A-U and G-C pairs that exhibit particularly strong internucleotide correlations (for example, pairs with $\rho>0.04$ ). This approach is analogous to the strategy previously used to determine through-space tertiary interactions from RING-MaP data (Homan et al. 2014). However, few base pairs exhibit strong correlations, and this strategy is thus limited by low sensitivity. Most Watson-Crick pairs exhibit relatively weak correlations that overlap the $\rho$-distribution observed for random noninteracting nucleotide pairs, making it difficult to increase sensitivity without compromising specificity. We therefore developed a two-step algorithm that harnesses fundamental principles of RNA secondary structure to robustly distinguish true correlations from false-positive noise, yielding de novo base-pair detection with both high sensitivity (sens) and low false discovery rates (FDR).

Beginning from the set of all RING correlations between $\mathrm{A}-\mathrm{U}$ and $\mathrm{G}-\mathrm{C}$ nucleotides (Fig. $2 \mathrm{~A}$ ), we select the strongest correlations measured for each nucleotide (Fig. 2B; and see Materials and Methods). This filtering is motivated by the hypothesis that paired nucleotides should exhibit the strongest RINGs to their direct Watson-Crick pairing partner. In the second step, we filter out isolated A-U and G-C correlations, selecting only those correlations that are supported by other adjacent parallel correlations (Fig. 2C). This second step recognizes that base pairs do not occur in isolation but rather as duplexes containing multiple pairs. Parallel correlations that reproduce this inherent property of real RNA secondary structures are unlikely to arise by chance.

This two-step algorithm does a good job of directly identifying Watson-Crick-paired nucleotides from RING-MaP data. We recovered $15 \%, 49 \%$, and $43 \%$ of Watson-Crick base pairs in the TPP riboswitch, P546 domain, and RNase P structures, respectively (Fig. 3). Strikingly, we detected multiple base pairs in both duplexes of the RNase P pseudoknot (Fig. 3B, boxed). If we consider detection of at least two base pairs to represent positive detection of a duplex, the algorithm exhibits excellent sensitivity for detecting duplexes containing four or more canonical base pairs $(14 / 16=88 \%)$ and moderate sensitivity overall $(17 / 32=53 \%$ for duplexes containing two or more base pairs) (Fig. 3B). The lower sensitivity for detecting pairs in duplexes that are short or that contain $\mathrm{G}-\mathrm{U}$ pairs is consistent with reduced duplex stability, 


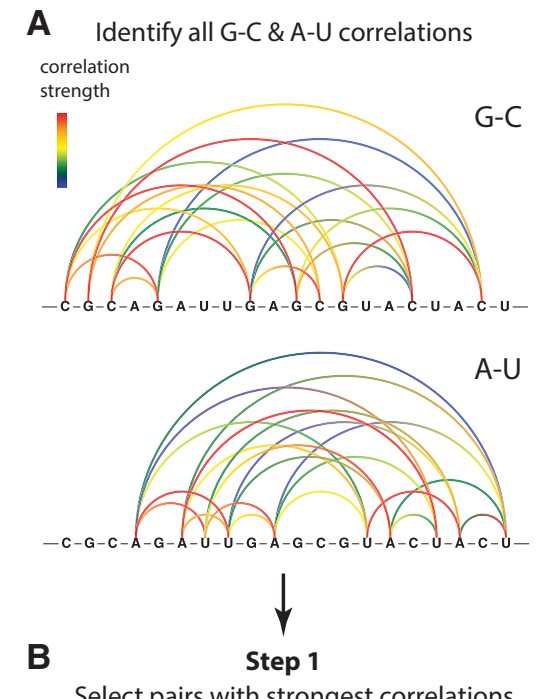

Select pairs with strongest correlations
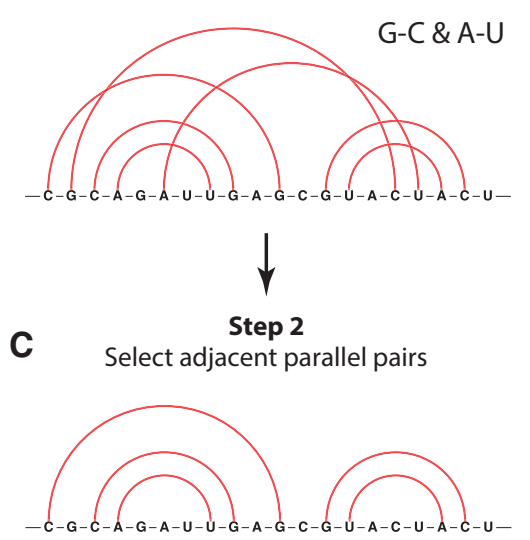

FIGURE 2. Two-step algorithm for detecting Watson-Crick RNA base pairs using RING-MaP correlated chemical probing data. (A) RING correlations are initially computed for all possible A-U and $\mathrm{G}-\mathrm{C}$ pairs. (B) In Step 1, the strongest correlation of each nucleotide is selected. (C) In Step 2, correlated pairs that are adjacent to other strongly correlated pairs are selected.

with nucleotides within such duplexes less likely to be modified in a correlated manner. Importantly, the false discovery rates were $0 \%, 10 \%$, and $0 \%$ for the TPP riboswitch, P546 domain, and RNase P, respectively. The two false-positive pairs identified in the P546 domain correspond to singlenucleotide register shifts adjacent to internal loops, which constitute minimal errors (Fig. 3B). Note that the ability to discriminate against false positives is critically dependent on filtering out isolated correlations (Fig. 3A). Thus, via simple filtering of RING-MaP data, we detect Watson-Crick base pairs de novo with high sensitivity and near-perfect specificity.

\section{Base-pair restraints enable high-accuracy secondary structure modeling}

The percentages of base pairs identified by our algorithm are insufficient to define complete RNA secondary structures.
We therefore explored the potential of using RING-MaPidentified base pairs to restrain thermodynamic secondary structure prediction algorithms. Each identified pair was given a small energy bonus $(-1 \mathrm{kcal} / \mathrm{mol})$ during minimum free modeling with the ShapeKnots algorithm of RNAstructure, which can predict pseudoknots (Hajdin et al. 2013).

For the TPP riboswitch and P546 domain, all RING-MaPidentified base pairs were predicted in the absence of data, and hence applying energy bonuses to these pairs did not improve secondary structure modeling (Supplemental Fig. S1). In the P546 domain, two incorrect RING-MaP-identified pairs were given energy bonuses, but the thermodynamic algorithm correctly distinguished the accepted pairing arrangement as more stable.

For RNase $\mathrm{P}$, base-pair bonuses dramatically increased modeling accuracy (Fig. 4). Whereas the RNase P model
TPP riboswitch

A Step 1 only

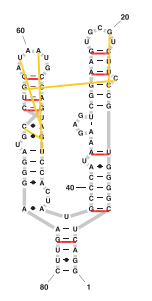

sens: $8 / 20=40 \%$ FDR: $5 / 13=38 \%$

B Step 1 and Step 2

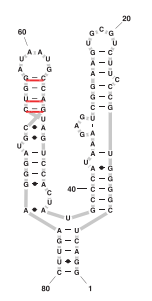

$\begin{aligned} \text { sens: } 3 / 20 & =15 \% \\ \text { FDR: } 0 / 3 & =0 \%\end{aligned}$
P546 domain

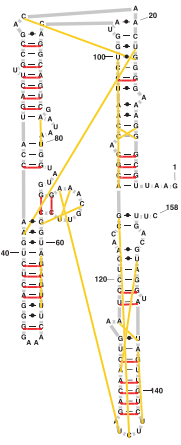

sens: $25 / 39=64 \%$ FDR: $13 / 38=34 \%$

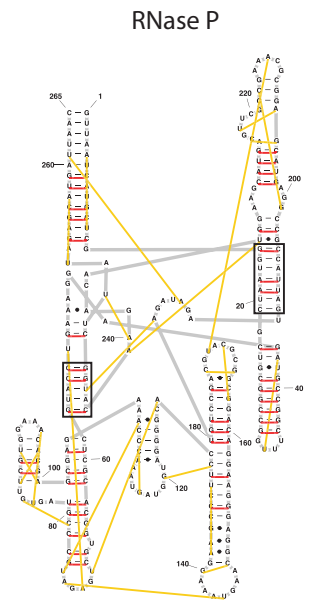

sens: $40 / 75=53 \%$ FDR: $24 / 64=38 \%$

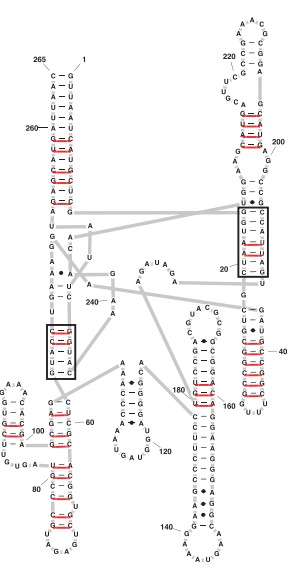

sens: $32 / 75=43 \%$

FDR: $0 / 32=0 \%$

FIGURE 3. Base pairs identified from RING-MaP data using $(A)$ only Step 1 of the algorithm, $(B)$ the complete two-step base-pair detection algorithm. Red and yellow lines connect correctly and incorrectly predicted base pairs, respectively, relative to accepted structures. The two helices that form the central pseudoknot in the RNase P RNA are boxed. 

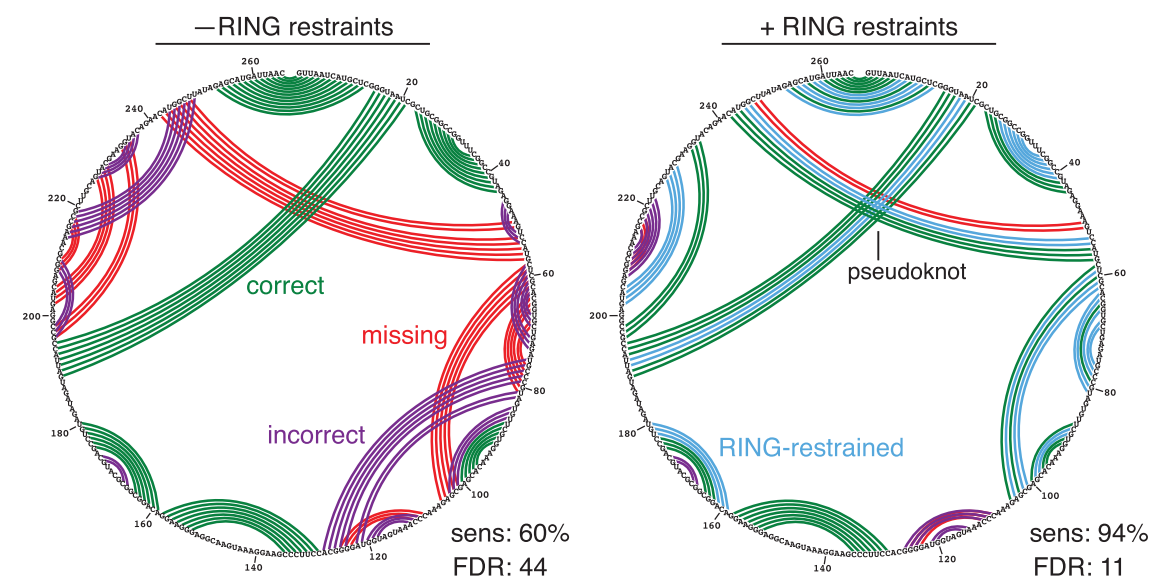

FIGURE 4. Secondary structure of RNase P catalytic domain predicted using thermodynamic minimum free energy modeling without and with RING-MaP restraints. Green arcs indicate correctly predicted pairs, purple arcs are incorrectly predicted pairs, and red arcs are true pairs missing in the predicted model. Blue arcs indicate enforced RING-MaP base-pairing restraints. although sensitivity depended strongly on read depth, the false discovery rate remained relatively constant at $0 \%-15 \%$. As above, the few false positives correspond to minor local register shift errors. Overall, this analysis indicates that, under the experimental conditions used here, read depths of $>200,000$ are necessary to enable base-pair detection at a level that is useful for secondary structure hypothesis generation.

\section{Increased experimental noise prevents base-pair detection in long RNAs}

To further evaluate our base-pair detection approach, we analyzed DMS RING$\mathrm{MaP}$ data collected on the E. coli $16 \mathrm{~S}$ and $23 \mathrm{~S}$ ribosomal RNAs (rRNAs). obtained from naïve secondary structure prediction misses the critical central pseudoknot, the RING-guided model recovered the pseudoknot and had an overall sens of $94 \%$ and FDR of $11 \%$, significantly outperforming the sens of $75.7 \%$ and the FDR of $20.2 \%$ reported in previous experiment-informed modeling studies (Hajdin et al. 2013). To our knowledge, this RING-guided model represents the first time the RNase P pseudoknot has been identified successfully in the absence of sequence covariation analysis. The errors in our RING-guided model are minor-a short extension of the pseudoknot is missed, several apical loops are modeled with additional pairs, and two duplexes exhibit minor register shifts (Fig. 4). In sum, the RING-guided model captures the essential features of the RNase P topology and demonstrates the potential of RING-MaP data for enabling high-accuracy secondary structure modeling.

\section{Relationship between base-pair detection and sequencing depth}

A key parameter in all sequencing-based structure-probing experiments is the minimum read depth required to obtain accurate measurements (Siegfried et al. 2014). Given that our base-pair detection algorithm depends on detecting correlations between weakly reactive nucleotides, we expected a strong dependence on read depth. We explored this dependence by varying the number of reads used in the correlational analysis for each RNA (Fig. 5; Supplemental Fig. S2). At the smallest tested read depth of 20,000, the sensitivity of our base-pair detection algorithm dropped to $\sim 10 \%$ for all three RNAs. At read depths of 200,000 , sensitivity increased to $>25 \%$, and 400,000 reads were needed to reliably detect the RNase P pseudoknot (Supplemental Fig. S2). This analysis suggests that low read depth is the source of the low sensitivity of base-pair detection in the TPP riboswitch. Importantly,
These rRNA data sets were obtained using a random-primed reverse-transcription strategy, in which individual MaP reads begin and terminate at random positions, tiling across each rRNA molecule. This contrasts with the directed-priming approach used for the shorter RNAs discussed above, for which each sequencing read extended across the entire RNA. Both the increased length (1542 nt for the 16S rRNA and 2904 nt for the $23 \mathrm{~S}$ rRNA) and random-primed strategy make base-pair detection significantly more challenging. Despite sequencing depths in excess of 1 million reads, our algorithm failed to identify any Watson-Crick base pairs in either the $16 \mathrm{~S}$ or $23 \mathrm{~S}$ rRNAs. Although this result represents a failure
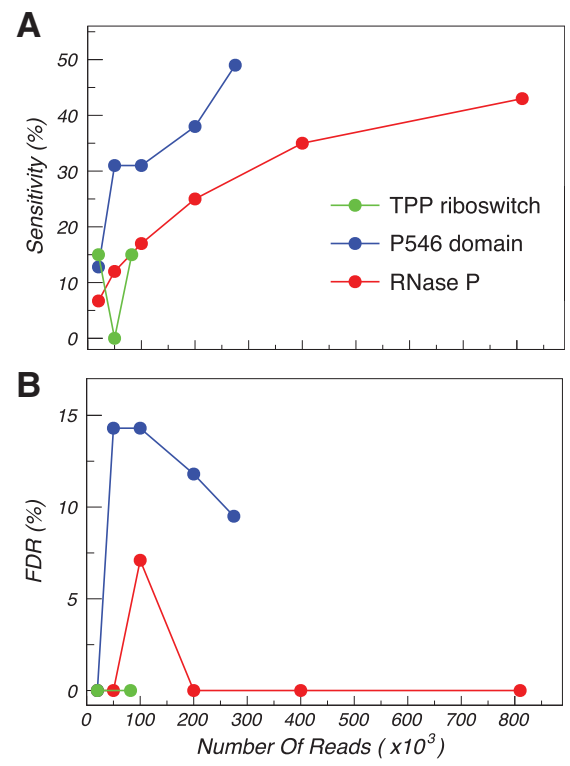

FIGURE 5. Accuracy of base-pair detection as a function of read depth. $(A)$ Sensitivity (sens) and (B) false discovery rate (FDR) are shown for the TPP riboswitch (green), P546 domain (blue), and RNase P (red) RNAs. 
with respect to base-pair detection sensitivity, the absence of false positives is a notable success and consistent with the low false discovery rate observed in the TPP riboswitch, P546 domain, and RNase P RNAs (Fig. 5). Thus, these analyses suggest that RING-based base-pair detection can be applied safely to diverse data sets without concern for false positives.

Detailed analysis of the $16 \mathrm{~S}$ and $23 \mathrm{~S}$ rRNA data sets revealed that, on average, Watson-Crick-paired nucleotides are more strongly correlated than random noninteracting nucleotide pairs ( $P$-value $<10^{-10}$ by the Kolmogorov-Smirnov test; Supplemental Fig. S3). However, base-paired nucleotides are much more weakly correlated than was observed in the shorter RNAs. There are several potential explanations for this reduction in correlation strength. First, the use of random priming during reverse transcription and the subsequent fragmented-library preparation may have introduced additional mutational noise relative to the directed-priming strategy used for shorter RNAs. Second, while the MaP protocol allows reverse-transcriptase enzymes to read-through modified nucleotides, stretches of RNA with multiple DMS modifications are less efficient templates for reverse transcription and more likely to experience premature termination. Co-mutation events may therefore be underrepresented in the randomly primed rRNA reads. In contrast, directed priming selects for full-length extension products, minimizing reverse-transcription biases. Together, increased mutational noise and reverse transcription biases may overwhelm the RING signal of Watson-Crick pairs in the rRNA data sets. Finally, the increased length, complex higher order structure, and conformational dynamics of rRNA molecules may introduce additional correlational relationships between nucleotides that mask those arising from Watson-Crick base pairs.

\section{DISCUSSION}

Upon reanalysis of prior RING-MaP data sets (Homan et al. 2014), we observed that DMS modification of $G$ and $U$ nucleotides can be detected via mutational profiling $(\mathrm{MaP})$. Based on these observations, we extend the RING-MaP strategy to enable direct identification of Watson-Crick base pairs. Studies of three representative model RNAs demonstrated that our approach has useful sensitivity and outstanding specificity, suggesting that RING-MaP experiments may be broadly useful as a strategy for directly visualizing RNA secondary structure.

Conceptually, our strategy shares some similarity to the mutate-and-map approach (Kladwang and Das 2010; Kladwang et al. 2011), which also detects correlated changes in nucleotide reactivity that occur between paired nucleotides. The mutate-and-map approach uses mutations to disrupt base pairs and requires collection of experimental data on hundreds of single-nucleotide variants. Thus, mutate-and-map offers high base-pair detection sensitivity, but requires substantially greater experimental overhead and is limited to synthetic RNAs. In contrast, the RING-MaP strategy requires a single DMS probing experiment on a single RNA, and can be performed on endogenous RNAs in living cells.

The primary limitations of our approach-modest sensitivity and high sequencing depth requirements-are functions of the low rate of DMS modification of $G$ and $U$ nucleotides (on average $<0.003$ ). Until now, DMS modification of $\mathrm{G}$ and $\mathrm{U}$ nucleotides has been largely undetectable and ignored. Two factors explain our ability to detect these DMS modifications. First, we use significantly higher reagent concentrations and improved buffer conditions to achieve modification rates that are an order of magnitude greater than those in typical DMS probing experiments (Homan et al. 2014). Second, the MaP strategy offers currently unparalleled detection sensitivity, allowing quantification of weak signals that would be indistinguishable from noise by traditional reverse-transcriptase termination-detection methods. While these experimental improvements allow us to reliably detect correlated modifications in $\mathrm{A}-\mathrm{U}$ and $\mathrm{G}-\mathrm{C}$ pairs in small RNAs, even modest degradation of data quality inhibits correlation detection in the $16 \mathrm{~S}$ and $23 \mathrm{~S}$ rRNAs. Developing improved reagents that react efficiently with all four nucleotides should alleviate these limitations, and thus stands as a transformative goal for future studies.

Even with improvements in sensitivity, we do not expect direct base-pair detection to recover 100\% of the base pairs in an RNA. Currently, we ignore G-U pairs due to a lack of sensitivity and high false-positive prediction rates, but these pairs are integral to many RNA helices. In addition, unstable base pairs may not exhibit correlated modifications and, conversely, base pairs that are highly stable may fail to give detectable modification signals. We demonstrate that incomplete sensitivity can be offset by using RING-MaP-identified base pairs to restrain thermodynamic secondary structure prediction algorithms. This combined approach mirrors the strategy used in both traditional SHAPE-directed modeling and mutate-and-map modeling. For RNase P, the combined approach worked particularly well, yielding a model that closely recapitulates the pseudoknotted accepted structure and significantly outperforming approaches that only use one-dimensional probing data (Fig. 4). Nevertheless, high accuracy structure modeling requires sufficient and dispersed RING restraints, with pseudoknot-spanning RINGs being particularly important for achieving an accurate RNase P model. We explored whether inclusion of one-dimensional DMS reactivity restraints could resolve the small errors in our RING-guided models, but this yielded negligible improvements in model accuracy (not shown). More sensitive one-dimensional probing data, such as that obtained from standard or differential SHAPE experiments (Rice et al. 2014), may prove more useful in further improving model accuracy.

Direct high-confidence identification of base pairs using a straightforward chemical probing experiment realizes a long- 
sought goal in RNA structure analysis. Our RING-MaPbased approach offers a powerful complement to existing structure probing and computational modeling strategies, and we envision key roles for RING-MaP analyses in both validation and de novo determination of RNA secondary structure.

\section{MATERIALS AND METHODS}

\section{Data collection and preprocessing}

Data for the E. coli thiamine pyrophosphate (TPP) riboswitch, the P546 domain of the T. thermophila group I intron, and the catalytic domain of B. stearothermophilus RNase P were collected previously (Homan et al. 2014). 16S and 23S rRNAs were isolated from E. coli K12 MG1655 cells at mid-log phase using nondenaturing conditions (Deigan et al. 2009). RNA was exchanged into a folding buffer containing $300 \mathrm{mM}$ sodium cacodylate $(\mathrm{pH} 7.0), 200 \mathrm{mM}$ potassium acetate $(\mathrm{pH} 7.0)$, and $10 \mathrm{mM} \mathrm{MgCl} 2$ using a gravity-flow column (PD-10 Sephadex, GE Healthcare), and incubated at $37^{\circ} \mathrm{C}$ for 20 min. DMS probing was performed at $37^{\circ} \mathrm{C}$ for 6 min exactly as previously described (Homan et al. 2014). Reverse transcription was performed using random nonamer primers (Smola et al. 2015) via an updated $\mathrm{MaP}$ protocol that includes the addition of betaine and stepped temperature cycles to improve reverse-transcriptase processivity (GM Rice and KM Weeks, in prep.). Sequencing libraries were prepared via random-primed tagmentation (Smola et al. 2015) and sequenced on an Illumina NextSeq instrument.

Sequencing data for the TPP riboswitch, P546 domain, and RNase P RNAs were trimmed, aligned, quality filtered, and converted to binary strings exactly as previously described (Homan et al. 2014). For the rRNA data sets, sequencing reads were pretrimmed using Sickle (https://github.com/najoshi/sickle), followed by alignment and quality filtering (Phred $>20$ ) using version 1.2 of the ShapeMapper pipeline (Siegfried et al. 2014). Filtered aligned reads were then converted into binary strings, with " 1 " representing mutated positions and "0" representing all other positions.

\section{Correlation analysis}

Correlations between nucleotides were computed using the covariance matrix

$$
\operatorname{cov}(i, j)=\frac{1}{N} \sum_{k=1}^{N}\left(n_{i}^{k}-\overline{n_{i}}\right)\left(n_{j}^{k}-\overline{n_{j}}\right),
$$

where $i$ and $j$ denote two positions in the RNA primary sequence, $k$ is the index of an individual sequencing read, $n_{i}^{k}\left(n_{j}^{k}\right)$ is the binary value of the read at position $i(j), N$ is the total number of sequencing reads, and $\bar{n}$ denotes the average over all reads. Pearson correlation coefficients $\rho_{\mathrm{ij}}$ were then computed from the covariance matrix

$$
\rho_{i j}=\frac{\operatorname{cov}(i, j)}{\sigma_{i} \sigma_{j}},
$$

where $\sigma_{i}\left(\sigma_{j}\right)$ is the standard deviation of $n_{i}\left(n_{j}\right)$. We note that for the dichotomous data considered here, $\rho_{\mathrm{ij}}$ are equivalent to $\phi_{\mathrm{ij}}$ values computed from contingency table analysis (as used in Homan et al. 2014). We also explored the potential of using partial correla- tions versus the ordinary correlations used here but observed no improvement in base-pair detection (not shown).

\section{Calculation of sensitivity and false discovery rate}

Sensitivity (sens) and false discovery rate (FDR) are defined as

$$
\begin{aligned}
& \text { sens }=\frac{T P}{T P+F N}, \\
& F D R=\frac{F P}{T P+F P},
\end{aligned}
$$

where TP (true positive) is the number of correctly identified A-U and $\mathrm{G}-\mathrm{C}$ base pairs, $F N$ (false negative) is the number of $\mathrm{A}-\mathrm{U}$ and $\mathrm{G}-\mathrm{C}$ pairs in the accepted RNA structure that are not identified by the algorithm, and FP (false positive) is the number of incorrectly predicted $\mathrm{A}-\mathrm{U}$ and $\mathrm{G}-\mathrm{C}$ pairs that do not exist in the accepted structure. No experimental data are available for the first three and last two nucleotides of the three short RNAs, and base pairs involving these nucleotides were excluded from sens and FDR calculations.

\section{Base-pair detection algorithm}

We identify A-U and C-G pairs using the following two-step algorithm (Fig. 2):

1. We identify tentative pairing partners by searching for complementary (A-U or $\mathrm{G}-\mathrm{C}$ ) nucleotides $i$ and $j$ that are more strongly correlated with each other than any other nucleotide in the molecule: $\rho_{i j} \geq \rho_{i k}$ and $\rho_{i j} \geq \rho_{l j}$, where $\rho_{i j}$ is the correlation coefficient between nucleotides $(i, j)$, and $k$ and $l$ are any other nucleotides in the sequence complementary to $i$ and $j$, respectively.

2. Correlated pairs identified in step 1 were then filtered by requiring that each $(i, j)$ correlation satisfy one of two selection rules: (a) another correlation exists at position $(i+1, j-1)$ or $(i-1$, $j+1$ ), or (b) two correlations exist at positions $(i+2, j-2)$ and $(i+3, j-3)$, or at positions $(i-2, j+2)$ and $(i-3, j+3)$ (see Fig. 2C). This filter eliminates isolated correlations.

\section{Secondary structure modeling}

Minimum free energy modeling was performed using the ShapeKnots algorithm of RNAstructure (v5.8) using default parameters (Hajdin et al. 2013). Base-pair energy bonuses were implemented by updating the ShapeKnots code to accept the "- $\mathrm{x}$ " experimental pair bonus option previously implemented in the Fold executable of RNAstructure (Kladwang et al. 2011). Each RING-MaP-identified pair was given a $-1 \mathrm{kcal} / \mathrm{mol}$ energy bonus, with all other pairs assigned bonuses of $0 \mathrm{kcal} / \mathrm{mol}$. Bonuses are singly applied to edge base pairs and doubly applied to internal base pairs. Additional modeling with one-dimensional DMS reactivity restraints was performed using the "-dms" option of ShapeKnots (Cordero et al. 2012). DMS reactivities were computed by subtracting the background mutation rate measured in no-reagent control samples (Homan et al. 2014) and normalizing as previously described (Deigan et al. 2009). Sensitivity and FDR calculations for minimum free energy models included G-U pairs and allowed for slipped base pairs (Deigan et al. 2009). 


\section{SUPPLEMENTAL MATERIAL}

Supplemental material is available for this article.

\section{ACKNOWLEDGMENTS}

This work was funded by the National Institutes of Health (GM 064803). A.M.M. is an Arnold O. Beckman Postdoctoral Fellow.

Received August 3, 2016; accepted October 28, 2016.

\section{REFERENCES}

Ashworth DJ, Baird WM, Chang CJ, Ciupek JD, Busch KL, Cooks RG. 1985. Chemical modification of nucleic acids. Methylation of calf thymus DNA investigated by mass spectrometry and liquid chromatography. Biomed Mass Spectrom 12: 309-318.

Bailor MH, Mustoe AM, Brooks CL III, Al-Hashimi HM. 2011. Topological constraints: using RNA secondary structure to model 3D conformation, folding pathways, and dynamic adaptation. Curr Opin Struct Biol 21: 296-305.

Bayfield MA, Dahlberg AE, Schulmeister U, Dorner S, Barta A. 2001. A conformational change in the ribosomal peptidyl transferase center upon active/inactive transition. Proc Natl Acad Sci 98: 10096-10101.

Cech TR, Steitz JA. 2014. The noncoding RNA revolution-trashing old rules to forge new ones. Cell 157: 77-94.

Chang C-J, Lee C-G. 1981. Chemical modification of ribonucleic-acid - a direct study by C-13 nuclear magnetic-resonance spectroscopy. Biochemistry 20: 2657-2661.

Cordero P, Kladwang W, VanLang CC, Das R. 2012. Quantitative dimethyl sulfate mapping for automated RNA secondary structure inference. Biochemistry 51: 7037-7039.

Deigan KE, Li TW, Mathews DH, Weeks KM. 2009. Accurate SHAPEdirected RNA structure determination. Proc Natl Acad Sci 106: 97-102.

Duncan CDS, Weeks KM. 2008. SHAPE analysis of long-range interactions reveals extensive and thermodynamically preferred misfolding in a fragile group I intron RNA. Biochemistry 47: 8504-8513.

Ehresmann C, Baudin F, Mougel M, Romby P, Ebel JP, Ehresmann B. 1987. Probing the structure of RNAs in solution. Nucleic Acids Res 15: 9109-9128.

Hajdin CE, Ding F, Dokholyan NV, Weeks KM. 2010. On the significance of an RNA tertiary structure prediction. RNA 16: 1340-1349.

Hajdin CE, Bellaousov S, Huggins W, Leonard CW, Mathews DH, Weeks KM. 2013. Accurate SHAPE-directed RNA secondary structure modeling, including pseudoknots. Proc Natl Acad Sci 110: $5498-5503$.

Homan PJ, Homan PJ, Favorov OV, Lavender CA, Kursun O, Ge X, Busan S, Dokholyan NV, Weeks KM. 2014. Single-molecule correlated chemical probing of RNA. Proc Natl Acad Sci 111: 13858-13863.

Kladwang W, Das R. 2010. A mutate-and-map strategy for inferring base pairs in structured nucleic acids: proof of concept on a DNA/RNA helix. Biochemistry 49: 7414-7416.
Kladwang W, VanLang CC, Cordero P, Das R. 2011. A two-dimensional mutate-and-map strategy for non-coding RNA structure. Nat Chem 3: 954-962.

Konforti BB, Liu Q, Pyle AM. 1998. A map of the binding site for catalytic domain 5 in the core of a group II intron ribozyme. EMBO J 17: $7105-7117$.

Krokhotin A, Houlihan K, Dokholyan NV. 2015. iFoldRNA v2: folding RNA with constraints. Bioinformatics 31: 2891-2893.

Kuśmierek JT, Singer B. 1976. Sites of alkylation of poly(U) by agents of varying carcinogenicity and stability of products. Biochim Biophys Acta 442: 420-431.

Lawley PD, Shah SA. 1972. Methylation of ribonucleic acid by the carcinogens dimethyl sulphate, $\mathrm{N}$-methyl-N-nitrosourea and N-methyl- $\mathrm{N}^{\prime}$-nitro-N-nitrosoguanidine. Comparisons of chemical analyses at the nucleoside and base levels. Biochem J 128: 117-132.

Leonard CW, Hajdin CE, Karabiber F, Mathews DH, Favorov OV, Dokholyan NV, Weeks KM. 2013. Principles for understanding the accuracy of SHAPE-directed RNA structure modeling. Biochemistry 52: 588-595.

Lorenz R, Bernhart SH, Höner Zu Siederdissen C, Tafer H, Flamm C, Stadler PF, Hofacker IL. 2011. ViennaRNA Package 2.0. Algorithms Mol Biol 6: 26.

Magnus M, Matelska D, Lach G, Chojnowski G, Boniecki MJ, Purta E, Dawson W, Dunin-Horkawicz S, Bujnicki JM. 2014. Computational modeling of RNA 3D structures, with the aid of experimental restraints. RNA Biol 11: 522-536.

Mortimer SA, Kidwell MA, Doudna JA. 2014. Insights into RNA structure and function from genome-wide studies. Nat Rev Genet 15: 469-479.

Peattie DA, Gilbert W. 1980. Chemical probes for higher-order structure in RNA. Proc Natl Acad Sci 77: 4679-4682.

Reuter JS, Mathews DH. 2010. RNAstructure: software for RNA secondary structure prediction and analysis. BMC Bioinformatics 11: 129.

Rice GM, Leonard CW, Weeks KM. 2014. RNA secondary structure modeling at consistent high accuracy using differential SHAPE. RNA 20: 846-854.

Seetin MG, Mathews DH. 2012. RNA structure prediction: an overview of methods. Methods Mol Biol 905: 99-122.

Siegfried NA, Busan S, Rice GM, Nelson JA, Weeks KM. 2014. RNA motif discovery by SHAPE and mutational profiling (SHAPE-MaP). Nat Methods 11: 959-965.

Sloma MF, Mathews DH. 2015. Improving RNA secondary structure prediction with structure mapping data. Methods Enzymol 553: 91-114.

Smola MJ, Rice GM, Busan S, Siegfried NA, Weeks KM. 2015. Selective 2 '-hydroxyl acylation analyzed by primer extension and mutational profiling (SHAPE-MaP) for direct, versatile and accurate RNA structure analysis. Nat Protoc 10: 1643-1669.

Weeks KM. 2010. Advances in RNA structure analysis by chemical probing. Curr Opin Struct Biol 20: 295-304.

Weeks KM. 2015. Toward all RNA structures, concisely. Biopolymers 103: $438-448$.

Weidmann CA, Weidmann CA, Mustoe AM, Weeks KM. 2016. Direct duplex detection: an emerging tool in the RNA structure analysis toolbox. Trends Biochem Sci 9: 734-736.

Zuker M. 2003. Mfold web server for nucleic acid folding and hybridization prediction. Nucleic Acids Res 31: 3406-3415. 

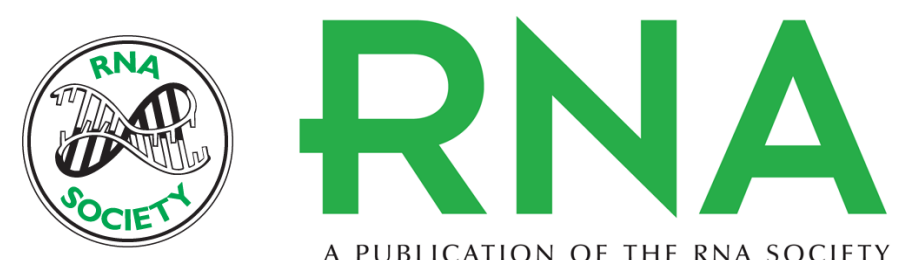

A PUBLICATION OF THE RNA SOCIETY

\section{Direct identification of base-paired RNA nucleotides by correlated chemical probing}

Andrey Krokhotin, Anthony M. Mustoe, Kevin M. Weeks, et al.

RNA 2017 23: 6-13 originally published online November 1, 2016

Access the most recent version at doi:10.1261/rna.058586.116

\section{Supplemental http://rnajournal.cshlp.org/content/suppl/2016/11/01/rna.058586.116.DC1 Material}

References This article cites 33 articles, 9 of which can be accessed free at: http://rnajournal.cshlp.org/content/23/1/6.full.html\#ref-list-1

Creative This article is distributed exclusively by the RNA Society for the first 12 months after the Commons License full-issue publication date (see http://rnajournal.cshlp.org/site/misc/terms.xhtml). After 12 months, it is available under a Creative Commons License (Attribution-NonCommercial 4.0 International), as described at http://creativecommons.org/licenses/by-nc/4.0/.
Email Alerting Receive free email alerts when new articles cite this article - sign up in the box at the Service top right corner of the article or click here.

\section{IIIII!' Providing Precise Solutions for your research.}

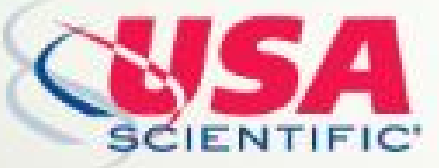

To subscribe to $R N A$ go to:

http://rnajournal.cshlp.org/subscriptions

(C) 2016 Krokhotin et al.; Published by Cold Spring Harbor Laboratory Press for the RNA Society 